\title{
An All-Hex Meshing Strategy for Bifurcation Geometries in Vascular Flow Simulation
}

\author{
Chaman Singh Verma ${ }^{1}$, Paul F. Fischer ${ }^{1}$, Seung E. Lee ${ }^{2}$, and F. Loth ${ }^{3}$ \\ 1 Mathematics and Computer Science Division, Argonne National Laboratory, \\ Argonne, IL 60439 \\ 2 Dept. of Mechanical Engineering, Massachusetts Institute of Technology, \\ Cambridge, MA 02319 \\ 3 Dept. of Mechanical Engineering, University of Illinois, Chicago, IL 60607
}

\begin{abstract}
Summary. We develop an automated all-hex meshing strategy for bifurcation geometries arising in subject-specific computational hemodynamics modeling. The key components of our approach are the use of a natural coordinate system, derived from solutions to Laplace's equation, that follows the tubular vessels (arteries, veins, or grafts) and the use of a tripartitioned-based mesh topology that leads to balanced high-quality meshes in each of the branches. The method is designed for situations where the required number of hexahedral elements is relatively small $(\sim 1000$ 4000 ), as is the case when spectral elements are employed in simulations at transitional Reynolds numbers or when finite elements are employed in viscous dominated regimes.
\end{abstract}

\section{Introduction}

We develop an automated all-hex meshing strategy for bifurcation geometries arising in subject-specific computational hemodynamics modeling. Our strategy is designed for the spectral element method (SEM) but works equally well for hex-based finite volume or finite element methods. Similar to the finite element method, the SEM is a high-order weighted residual technique featuring isoparametric hexahedral (hex) elements that are globally assembled into an unstructured mesh. A distinguishing feature of spectral element meshes is that they generally require orders of magnitude fewer elements than their finite-element counterparts because each spectral element typically contains 100 s to 1000 s of gridpoints. This reduction often poses significant challenges for conventional approaches to automated hex-mesh generation because there are relatively few interior elements over which to apply smoothing in order to absorb topological corrections that arise from, say, merging advancing fronts. It is of interest, therefore, to construct meshes having intrinsically compatible geometries and topologies. For most hemodynamic flow domains, which primarily comprise tubes and bifurcations, there are decompositions that are 
readily tessellated by hexahedra using sweeping methods $[8,9]$. The challenge, however, is to develop robust, fast, and fully automated schemes for patient-specific geometries, which often feature tortuous passages with sharp curvature and (in the presence of stenoses) rapid variation in diameter. An additional complication, separate from the question of meshing is that the geometry is not well defined but must generally be inferred from slice-based medical images that are often highly pixelated with respect to the vessel diameter. Certainly, the geometry definition, which involves image registration and segmentation, is an integral part of the automated procedure, but we do not discuss it further here. We also note that, while the mesh generation techniques proposed here are designed specifically for vascular flow geometries, they could be adapted to work equally well in other internal flow configurations involving bifurcating channels.

\section{Background and Flow Modeling}

During the past two decades, the role of hemodynamics, or fluid mechanics of blood flow, has been implicated in the development of arterial disease and in the regulation of cellular biology in both normal and diseased arteries $[5,6]$. Vascular disease, including atherosclerosis, aneurysms, and plaque disruption is one of the leading causes of death in the United States. A number of methods are being used to investigate the hemodynamic forces in the vascular system, with computational fluid dynamics (CFD) becoming the most prevalent because of its ability to provide more detailed flow information than either in vivo or in vitro experiments. Although significant insight has been gained from CFD simulations in idealized vascular geometries, geometry clearly has a dominant influence on the local hemodynamics and there is a consequent need for subject-specific vascular flow modeling [7].

While the natural flow state in the vasculature is laminar, it is possible to have a transition to a weakly turbulent state in the presence of stenoses (blockages) or subsequent to surgical procedures such as arteriovenous graft implantation [12]. The transition to turbulence induces a sudden change in the range of spatial and temporal scales in the solution, resulting in a need for two to three orders of magnitude increase in computational resources for the same physical-time simulation. The flow physics in the turbulent case is dominated by convection of momentum with relatively little diffusion. The non-dimensional ratio of these two processes is denoted as the Reynolds number, which is typically $R e \sim 350$ in healthy vessels but can reach as high as $R e=1000-3000$ in the transitional cases. For simulations in the high Reynolds number regime where physical dissipation is small, it is beneficial to use high-

order numerical discretizations that have minimal numerical dispersion and dissipation per grid point [2].

Our numerical approach is based on the SEM, which is a high-order weighted residual technique that combines the geometric flexibility of the 
finite element method (FEM) with the rapid convergence and tensor-product efficiencies of global spectral methods. In the SEM, the domain is decomposed into $E$ curvilinear hexahedral elements, and the solution within each element is represented as an $N$ th-order tensor-product nodal-based (Lagrange) polynomial. For three-dimensional problems, there are approximately $E N^{3}$ gridpoints in the entire domain.

We remark that high-order methods do not circumvent the need to resolve flow structures such as boundary layers and vortices. Both low- and highorder methods must capture the predominant structures. In the FEM the requisite resolution is attained by varying $E$, whereas in the SEM the resolution is attained through a combined variation of $E$ and $N$, with $N=8-12$ being typical. For the same resolution (i.e., number of gridpoints) the number of elements for an SEM discretization would be two to three orders of magnitude smaller than its FEM counterpart. For example, typical SEM discretizations of a bifurcation geometry involve 1000-4000 elements, whereas typical FEM discretizations involve $>10^{5}$ elements. Because there are so few elements, it is critical that the SEM mesh topology and geometry be relatively free of the point and line dislocations that can result when two or more mesh fronts converge when using advancing front or similar generalized meshing methods. For the FEM, achieving this topology is less of a problem because mesh smoothing can be used to effectively spread the geometric penalty arising from such topological defects over a large number of elements. For the SEM, mesh smoothing is also important but is more constrained by the (topological) proximity of the boundaries. Fortunately, for the class of geometries that we are considering here, which comprises blood vessels and bifurcations, high-quality all-hex decompositions exist, and we are able to exploit this domain-specific information to develop a robust and fully automated meshing procedure that works equally well for the SEM and, through mesh refinement, for the FEM.

\section{Vessel Surface Definition}

Currently, we are using noncompact radial basis functions (RBFs) to represent the vessel surface [1]. We opted for noncompact RBF for the following reasons: (i) the initial point cloud was noise-free, (ii) the number of points was reasonable to be handled by ordinary Linux machines, and (iii) noncompact thin-plate RBFs provide smooth surfaces. The RBF-based implicit surface construction requires a set of known interpolating points that we construct as follows. Given surface points $\mathbf{x}_{j}, j=1, \ldots, n$, we identify a set of external points, $y_{k}, k=1, \ldots, m$, near the surface using an oct-tree algorithm that marches inward with successively smaller boxes from the edges of a bounding box. With the known oct-tree topology, we identify as external points the centers of small empty boxes adjacent to those containing surface points. From these, we define a signed distance and construct an interpolating RBF, $\phi(\mathbf{x})$, 
that vanishes at all $\mathbf{x}_{j}$ and matches the signed distance at all $y_{k}$. The surface where $\phi(\mathbf{x}) \equiv 0$ defines the vessel wall.

The radial basis function is evaluated on a set of structured points, and the $\phi=0$ surface is then triangulated by using the marching cubes algorithm [11]. This surface triangulation is then smoothed by using Taubin's nonshrinking smoothing algorithm $[17,18]$. It was observed that RBF-based surface is not accurate near the end-caps. These sites are locations where artificial boundary conditions are applied in the blood-flow simulations and are thus not required to be physically correct. We therefore cut the end-caps and triangulated the surface cuts using Shewchuck's Triangle software [15].

\section{Conduction-Based Sweeping}

To introduce the basic elements of our bifurcation meshing procedure, we consider a simpler model problem, namely, the meshing of a curvilinear tube representative of an artery or vein. The strategy is to employ a sweeping algorithm, in which one projects a templated quadrilateral $O$-grid onto specified cross-sections of the tube and connects corresponding vertices in adjacent cross-sections to form a hex mesh. The central element to our meshing strategy is to develop a natural coordinate system that follows the undulating vessel and satisfies two criteria:

Criterion 1: no two cross-sections intersect, and

Criterion 2: each cross-section is orthogonal to the vessel wall.

A robust approach to producing such a coordinate system is to solve Laplace's equation in the vessel with Dirichlet boundary conditions at the tube ends and homogeneous Neumann conditions along the tube wall. This corresponds to a steady thermal conduction problem with, say, temperature $\alpha=0$ at one end, $\alpha=1$ at the other, and $\nabla \alpha \cdot \hat{\mathbf{n}}=$ along the sidewalls, where $\hat{\mathbf{n}}$ is the outward pointing normal. Once $\alpha$ is known, selecting a set of isosurfaces for a monotonically increasing sequence of temperatures $0=\alpha_{1}<\alpha_{2}<\ldots<$ $\alpha_{m}=1$ will produce a set of cross-sections that satisfy the desired nonintersecting and orthogonality conditions. These surfaces often have double curvature like a potato chip and are therefore termed chips. The $\alpha_{i}$ s can be chosen such that chips have the desired separation in Euclidean space. A typical set of chips and a 12-element $O$-grid template are shown in Fig. 1.

We note that potential-based grid generation is a well-established technique [19], particularly for external flow configurations, where analytical solutions to Laplace's equation can readily be computed. In the present case, one needs a base mesh in order to solve the conduction (i.e., potential) problem. Fortunately, Laplace's equation is very well conditioned and virtually any reasonable mesh/discretization pairing is up to the task, particularly because we are not concerned with accuracy but only with satisfying the non-intersection and orthogonality criteria. We therefore employ linear finite elements for the 


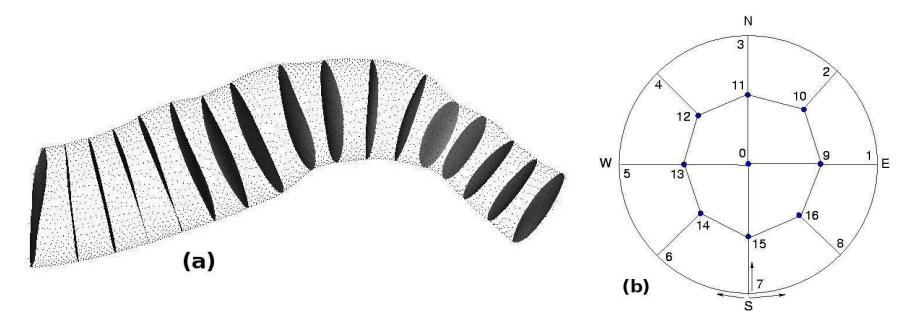

Fig. 1. (a) Isosurfaces of $\alpha$ in an isolated vessel. (b) Level-0 $O$-grid template.

conduction problem. Starting with a surface triangulation, we build a tetrahedral mesh using the advancing front algorithm [13]. We then construct the finite element Laplacian, apply the requisite boundary conditions, and solve the sparse linear system by using conjugate gradient iteration. The surface triangulation is smoothed by using Taubin's non-shrinking algorithm [17]. With $\alpha$ known, the $O$-grid is swept through the domain and projected onto chips where $\alpha(\mathbf{x})=\alpha_{i}$. The $O$-grid template is aligned by minimizing the Euclidian distance between the "S" points (Fig. 1b) when the template is mapped onto adjacent chips. The remaining edge points are distributed according to arclength. The interior point distribution is parameterized by using local polar coordinates, with arclength acting as the azimuthal variable, and the radial coordinate normalized by the distance from the chip centroid to the perimeter.

\section{Bifurcation Geometries}

Our bifurcation meshing strategy is based on a tripartition of the domain into three tubes, each of which is meshed using a variant of the sweeping algorithm described in the preceding section. This approach yields minimal elemental deformation and balanced resolution in each branch. (The initial mesh can be further improved through mesh smoothing techniques $[4,14]$ and the local resolution enhanced through $p$ - or $h$-type refinement, e.g., [3].) Our original implementation of the tripartition algorithm was based on a series of cross-sections that were orthogonal to the plane containing the bifurcation $[8,9]$. Such an approach, however, is not robust because it does not guarantee Criteria 1 and 2. We therefore extend this tripartition approach to incorporate the natural coordinate system introduced in Section 4.

We demonstrate that, in addition to satisfying Criteria 1 and 2, the Laplacian isosurfaces provide a robust and natural tripartition of the bifurcation geometry. We begin by noting that any two of the three branches may be swept by solving Laplace's equation with $\alpha=0$ on one end-cap, $\alpha=1$ on another, and $\nabla \alpha \cdot \hat{\mathbf{n}}=0$ elsewhere. The third branch can be swept by cyclically permuting these boundary conditions and solving a second conduction problem. For symmetry and for completeness of the algorithm, we solve three conduction problems in total, each having, in turn, one branch insulated while 
Table 1. Boundary Conditions for Bifurcation Conduction Problems

\begin{tabular}{lllll} 
Problem & $\Gamma_{A}$ & $\Gamma_{B}$ & $\Gamma_{C}$ \\
\hline$A:$ & $\nabla^{2} \alpha=0$ & $0=\nabla \alpha \cdot \hat{\mathbf{n}}$ & $\alpha=-\alpha_{P}$ & $\alpha=1-\alpha_{P}$ \\
$B:$ & $\nabla^{2} \beta=0$ & $\beta=1-\beta_{P}$ & $0=\nabla \beta \cdot \hat{\mathbf{n}}$ & $\beta=-\beta_{P}$ \\
$C:$ & $\nabla^{2} \gamma=0$ & $\gamma=-\gamma_{P}$ & $\gamma=1-\gamma_{P}$ & $0=\nabla \gamma \cdot \hat{\mathbf{n}}$
\end{tabular}

the other two have end-caps at different fixed values. Denoting the branches as $A, B$, and $C$, with respective end-caps $\Gamma_{A}, \Gamma_{B}$, and $\Gamma_{C}$, we solve conduction problems for $\alpha, \beta$, and $\gamma$ satisfying the boundary conditions listed in Table 1. For each case, the Dirichlet values have been shifted by a constant (denoted with subscript $P$ ) so that the temperature at the center of the insulated endcap is zero. The isosurfaces that (nominally) emanate from these centerpoints are referred to as the principal isosurfaces and, with the shift, correspond to $\alpha=0, \beta=0$, and $\gamma=0$.

The solutions for problems $A-C$ are shown in Fig. 2 for a stenosed carotid artery bifurcation. A critical observation is that, to the level of truncation error, the principal isosurfaces intersect in a unique curve, as illustrated in Fig. 2d. (Uniqueness is further discussed in Appendices A and B.) These isosurfaces consequently serve to tripartition the bifurcation, as required for our sweeping strategy. In addition, they provide the bisecting "plane" that is required to orient the $O$-grid so that element boundaries are aligned with the cusp of each branch where it connects to the bifurcation. Thus six subdomains are defined by the principal isosurfaces: three branches, each having two halves. Denoting the half of branch $A$ that connects to $B$ by $A_{B}$, and so on for each half, we formally identify "half-chips" in subdomains $A_{B}$ and $B_{A}$ with isosurfaces of $\gamma$, those in $B_{C}$ and $C_{B}$ with isosurfaces of $\alpha$, and those in $C_{A}$ and $A_{C}$ with isosurfaces of $\beta$. As in Section 4, the chips defining adjacent slabs of hex elements are found by choosing $\alpha$-isosurfaces (or $\beta$ - or $\gamma$-) that yield a desired Euclidean separation. (Hereon, unless otherwise indicated, we will restrict our discussion to the problem of meshing the $A$-branch using isosurfaces of $\beta$ and $\gamma$, with the understanding that the procedure for the other branches follows from symmetry.)

\section{Refinements to the Approach}

In infinite-precision arithmetic, the approach outlined above would suffice to mesh each branch in its entirety. A modest complication arises because the insulated branch assumes a nearly uniform temperature as one moves away from the bifurcation. Choosing $\alpha_{P}$ so that the temperature in the insulated branch is close to zero and then recomputing $\alpha$ provides several significant digits that would otherwise be lost and allows one to more precisely identify the principal isosurface. Nevertheless, it is often difficult to identify the principal isosurface more than two diameters away from the bifurcation, as is clear 
from Fig. 2. For two reasons, this situation is not a particular problem. First, we note that identifying the principal isosurface near the bifurcation is a very robust procedure. In exact arithmetic, all isosurfaces in the insulated branch collapse exponentially fast to the principal isosurface as one approaches the bifurcation. Thus, starting from any point in the insulated branch, one can use a marching tetrahedra algorithm and arrive at the principal isosurface. Second, away from the bifurcation, the principal isosurface merely serves as a guide to orient the $O$-grid and is not really needed. In the extremal regions, the $O$-grid can be mapped to either constant- $\beta$ or constant- $\gamma$ surfaces (for the $A$-branch), as the two families are indistinguishable. Thus, away from the bifurcation, we simply pick one of two solutions (e.g., $\beta$ or $\gamma$ ) and sweep toward the extremity $(A)$ following the procedure of Section 4 .

\section{Summary of Approach}

With the basic concepts in hand, we summarize the steps of the procedure as follows:

1. Starting from a noncompact RBF representation, construct a triangulation of the vessel surface.

2. Apply Taubin smoothing [17] to the surface triangulation.

3. Use the advancing front algorithm to construct a preliminary tet mesh of the domain volume.

4. Using linear finite elements and conjugate gradient iteration, solve conduction problems for $\alpha, \beta$, and $\gamma$ (Table 1) with $\alpha_{P}=\beta_{P}=\gamma_{P}=0$.

5. Set $\alpha_{P}, \beta_{P}$, and $\gamma_{P}$ to the negative of their respective insulated endcap centerpoint values and recompute $\alpha, \beta$, and $\gamma$. (Iterate on Step 5 , if needed.)

6. Identify the $\alpha=0$ principal isosurface with marching tets, and compute $\beta$ and $\gamma$ on this surface using the corresponding marching-tet interpolants.

7. Use marching triangles to identify the $\beta=0$ and $\gamma=0$ curves on the $\alpha=0$ principal isosurface. Average these curves to determine a common representation of the trisection curve. Identify the centerpoint (measured in arc-length) of the trisection curve as the origin, $O$.

8. On the $A$-branch, march outward from $O$ on the $\alpha=0$ surface a userspecified distance $\delta$ (typ., $\delta \approx 1 / 4 D$, where $D$ is the local vessel diameter). Denote the corresponding point as $\mathbf{x}_{1}$.

9. Find the values $\beta_{1}:=\beta\left(\mathbf{x}_{1}\right)$ and $\gamma_{1}:=\gamma\left(\mathbf{x}_{1}\right)$, and compute the corresponding isosurfaces $\beta(\mathbf{x})=\beta_{1}$ and $\gamma(\mathbf{x})=\gamma_{1}$ with marching tets. Identify the first pair of half-chips with these isosurfaces.

10. Repeat Steps 8 and 9 , generating points $\mathbf{x}_{i}$ and corresponding isosurfaces until the dihedral angle of the $\beta(\mathbf{x})=\beta_{i}$ and $\gamma(\mathbf{x})=\gamma_{i}$ chips at $\mathbf{x}_{i}$ is $<10^{\circ}$; then switch to the single-chip algorithm of Section 4 using either $\beta$ or $\gamma$ as the coordinate.

11. Starting at $O$, project templated $O$-grids onto each chip or half-chip pair. 

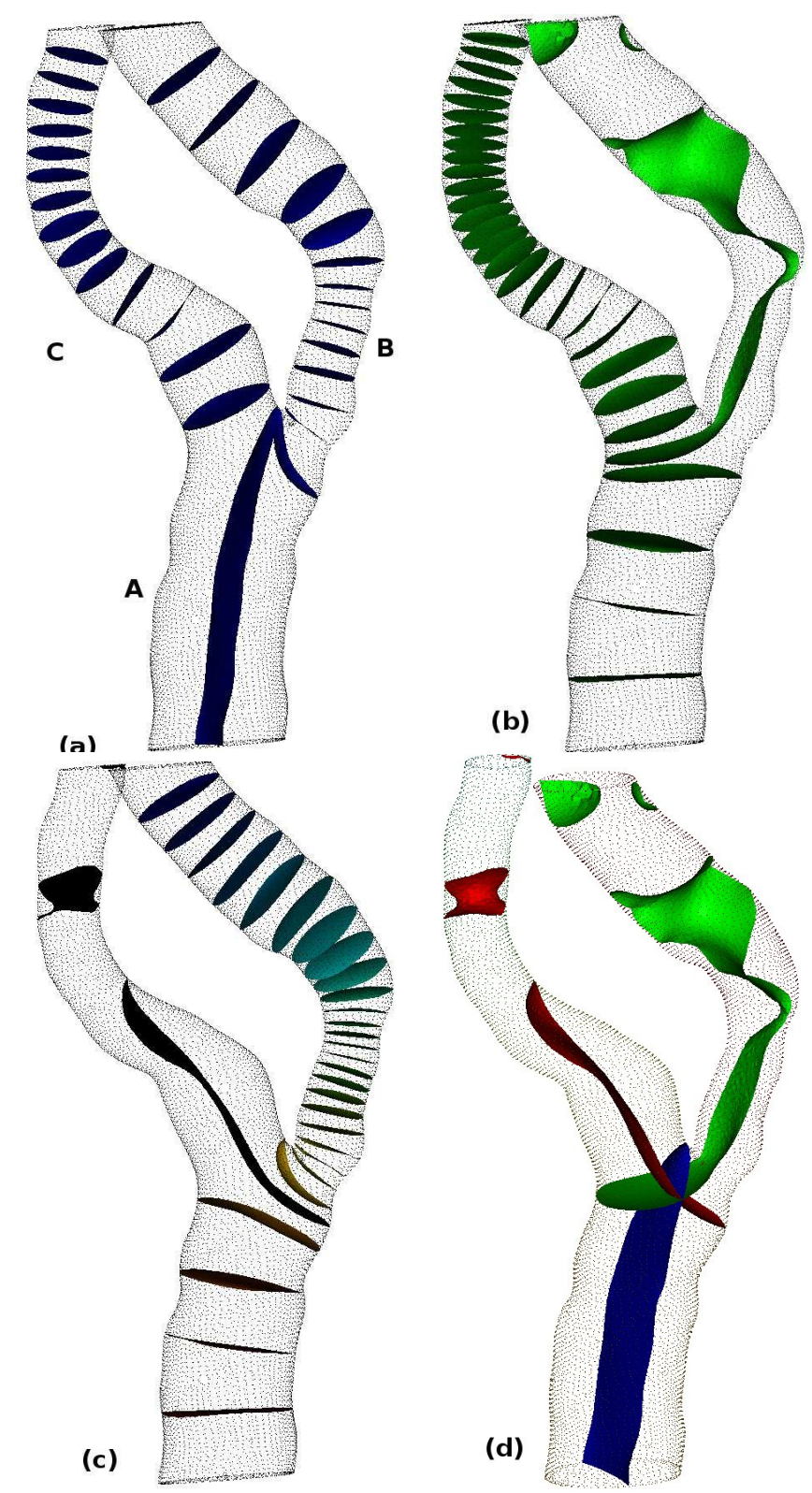

Fig. 2. Isosurfaces for conduction problems $A$ (a), $B$ (b), and $C$ (c) of Table 1; (d) intersection of principal isosurfaces. 

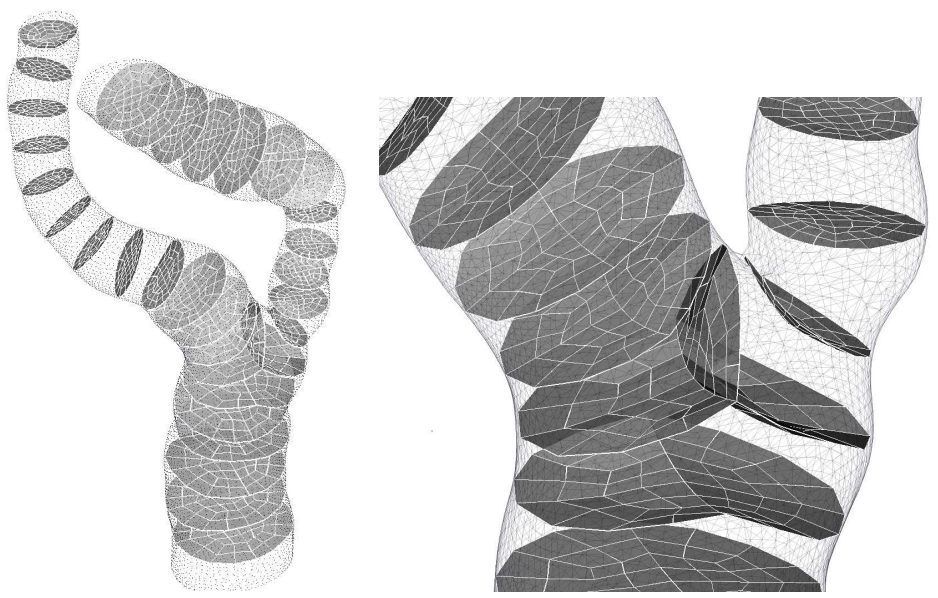

Fig. 3. Templated chips in a bifurcation geometry.

12. Smooth the quadrilateral surface mesh using Taubin smoothing, and project the smoothed points onto the triangulated chip surface.

13. Repeat Steps 8-12 for branches $B$ and $C$.

14. Consistently order each contour on each chip with respect to the principal isosurface half-chips.

15. Construct the hexahedral volume mesh taking two adjacent chips at a time.

Figure 3 shows the resulting templated chip set for the stenosed carotid bifurcation. A close up of the half-chips on the principal isosurfaces that trisect the bifurcation are shown in Fig. 3b.

\section{Results}

We have successfully used an earlier variant of this approach, developed in $[8,9]$, to build several meshes for the study of transition in vascular flows $[10,12]$, This earlier mesh construction approach involved several disjoint pieces of software and required significant user expertise to produce an acceptable mesh. Typical turnaround time was on the order of five to ten days. Our current focus is on streamlining the entire procedure so that it is fully automated and requires minimal user input.

The current development code is in $\mathrm{C}++$ and uses the Matrix Template Library [16] to solve the linear equations for the RBF and heat conduction problems. The RBF coefficients were computed by using direct solves, whereas the conduction problems were solved with Jacobi-preconditioned conjugate gradient iteration. Table 2 shows preliminary performance results on a Linux $2.4 \mathrm{GHz}$ Pentium IV machine with a breakdown of the CPU time for each 
phase. We stress that this initial development code has not been optimized. The turnaround for the entire procedure has nonetheless been reduced to a matter of minutes, rather than days.

Table 2. Timing for Automated Generation of Carotid Bifurcation Mesh

\begin{tabular}{|l|c|}
\hline Function & Time ( in Seconds ) \\
\hline RBF coefficients evaluation & 450 \\
RBF function evaluation on box & 90.0 \\
Marching cube for $\phi=0$ evaluation & 2 \\
Taubin surface fairing & 1 \\
Trimming end sections & 0.5 \\
Solution of three heat equations & 400 \\
Principal chips construction & 1 \\
Split chips construction & 2 \\
Simple chips construction & 4 \\
Mesh template & 0.5 \\
Surface smoothing & 0.001 \\
Volume mesh & 0.0001 \\
\hline
\end{tabular}

\section{Future Directions}

Our goal is to provide rapid generation of a quality hex mesh for vascular flow geometries, starting with a sliced-based stack of medical images (i.e., CT-scan or MRI). presently, we are using commercial software to segment the images. This will be replaced by a snake algorithm that is incorporated as a module in our mesh generation software. With all the modules in place, we will tackle optimization of the algorithms described in the preceding sections. In particular, we expect significant performance gains by using multigrid to precondition the thermal conduction problems. Similar performance gains are expected by improving the RBF approach. For instance, we have been investigating the use of multilevel RBFs that yield sparse coefficient matrices.

\section{Acknowledgments}

This work was supported by the National Institutes for Health, RO1 Research Project Grant (2RO1HL55296-04A2) and by the Mathematical, Information, and Computational Sciences Division subprogram of the Office of Advanced Scientific Computing Research, U.S. Department of Energy, under Contract W-31-109-Eng-38. 

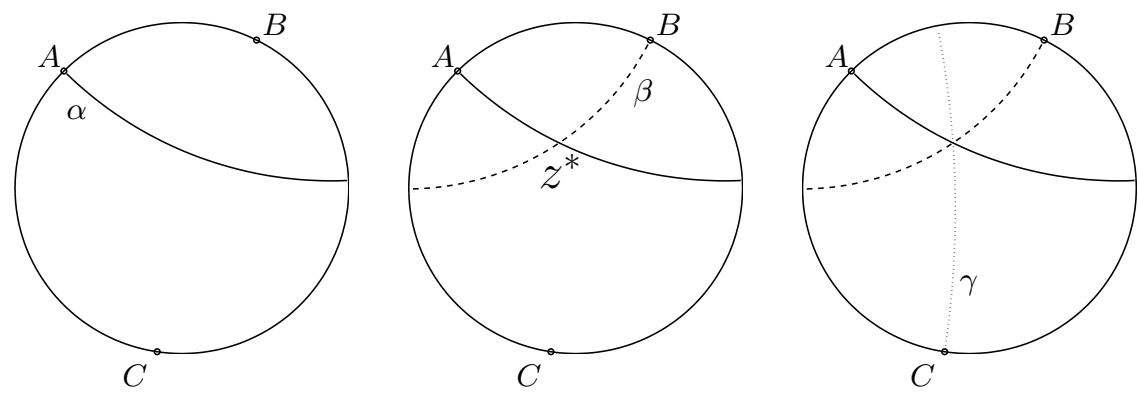

Fig. 4. Two-dimensional model problem showing common intersection point $z^{*}$ for isopotentials $\alpha, \beta$, and $\gamma$.

\section{Appendix A}

Our assertion that the three principal isosurfaces intersect in a unique onedimensional curve is based primarily on observation of tens of two- and threedimensional cases. Here, we demonstrate that uniqueness holds rigorously for a special class of two-dimensional geometries, namely, for an arbitrary distribution of source, sink, and isocontour points on a circle.

Consider the two-dimensional potential problem in the unit-disk having a point source at $B$ and a point sink of equal strength at $C$, as illustrated in Fig. 4. The general solution in the complex $z$-plane is

$$
u+i v=\ln \frac{z-B}{z-C}+c
$$

with $u$ and $v$ real-valued, $i:=\sqrt{-1}$, and $c$ arbitrary. The isopotentials (corresponding to isothermal surfaces in Fig. 2) are given by isocontours of the real part of the solution, $u$, and in this case are circular arcs. The isosurface passing through $A$ is given by

$$
\alpha:=\left\{z:\left|\frac{z-B}{z-C}\right|=\left|\frac{A-B}{A-C}\right|\right\} .
$$

Cyclically permuting the role of $A, B, C$ yields a new problem with isocontour $\beta$ passing through $B$ given by

$$
\beta:=\left\{z:\left|\frac{z-C}{z-A}\right|=\left|\frac{B-C}{B-A}\right|\right\} .
$$

The intersection of $\alpha$ and $\beta$ is given by the point $z^{*}$ that simultaneously satisfies (1) and (2). Inserting $z^{*}$, multiplying (1) and (2), and inverting the result shows that $z^{*}$ also satisfies

$$
\left|\frac{z^{*}-A}{z^{*}-B}\right|=\left|\frac{C-A}{C-B}\right|,
$$




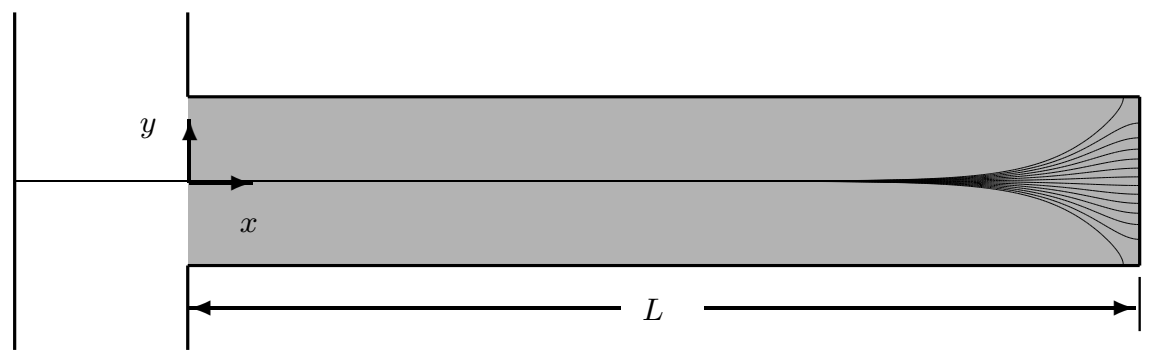

Fig. 5. Two-dimensional model problem illustrating exponential convergence of end-cap isosurfaces to the principal isosurface.

which implies that $z^{*}$ is also an element of $\gamma$, the third principal isocontour obtained by again permuting the roles of $A, B$, and $C$. Thus, the intersection of the principal isocontours $\alpha, \beta$, and $\gamma$ is unique.

Note that this class of solutions can be immediately extended to any domain $\Omega$ that whose boundaries are given by isocontours of $v$. Such boundaries are orthogonal to the isopotential lines and an arbitrary choice of these boundaries does not affect the solution $u$. Thus, the result is immediately applicable to football and crescent shaped domains. Numerical evidence suggests that the result holds for any simply connected domain in $\mathbb{R}^{2}$, but we've yet to rigorously establish this generalization.

\section{Appendix B}

The model problem of Appendix A employed idealized point sources and sinks to establish the unique intersection point of the three principal isosurfaces. In our meshing application, we have set the entire end-caps to be either a fixed temperature or insulated. Strictly speaking, we can no longer expect uniqueness of the intersection point because there is an infinity of isosurfaces emanating from the insulated end-cap. The following example, however, illustrates that these isosurfaces collapse exponentially fast to the principal isosurface. Consider the computational domain $\Omega$ with $(x, y) \in[0, L] \times\left[-\frac{1}{2}, \frac{1}{2}\right]$ indicated by the gray region in Fig. 5 , which is a two-dimensional model of an insulated branch having unit diameter $D$. To first order, we can approximate the potential at the left edge of the domain as $T(0, y)=\sin \pi y$, for which the solution is $T=\sin \pi y \cosh \pi(x-L) / \cosh \pi L$. An isosurface emanating from the right at $\left(L, y_{0}\right)$ is given by

$$
y=\sin ^{-1}\left[\frac{\sin \pi y_{0}}{\cosh \pi(L-x)}\right],
$$

which establishes the exponential convergence to the principal isosurface. Several such isosurfaces are shown in the figure. As an example, if $L=5 D$, then 
the maximum separation of any isosurface pair emanating from the end-cap is $<5 . e-7$ at the bifurcation point.

\section{References}

1. J.C. Carr, W.R. Fright, and R.K. Beatson, Surface interpolation with radial basis functions for medical imaging, IEEE Trans Med. Imaging 16 (1997), 96-107.

2. M.O. Deville, P.F. Fischer, and E.H. Mund, High-order methods for incompressible fluid flow, Cambridge University Press, Cambridge, 2002.

3. P.F. Fischer, G.W. Kruse, and F. Loth, Spectral element methods for transitional flows in complex geometries, J. Sci. Comput. 17 (2002), 81-98.

4. L. Freitag and P. Plassmann, Local optimization-based simplical mesh untangling and improvement, Int. J. Numer. Methods Eng. 48 (2000), 109-125.

5. M. H. Friedman, B. D. Kuban, P. Schmalbrock, K. Smith, and T. Altan, Fabrication of vascular replicas from magnetic resonance images, J. Biomech. Eng. 117 (1995), 364-366.

6. D. P. Giddens, C. K. Zarins, and S. Glagov, The role of fluid mechanics in the localization and detection of atherosclerosis, J. Biomech. Eng. 115 (1993), $588-594$.

7. D. N. Ku, Blood flow in arteries, Annu. Rev. Fluid Mech. 29 (1997), 399-434.

8. Piersol N. Loth F. Fischer P. Leaf G. Smith B. Yedevalli R. Yardimci A. Alperin N. Lee, S.E. and L. Schwartz, Automated mesh generation of an arterial bifurcation based upon in vivo mr images, Proceedings of the 2000 World Congress on Medical Physics and Bioengineering CD ROM (2000).

9. S.E. Lee, Solution method for transitional flow in a vascular bifurcation based on in vivo medical images, Master's thesis, Univ. of Illinois, Chicago, 2002, Dept. of Mechanical Engineering.

10. S.W. Lee, P.F. Fischer, F. Loth, T.J. Royston, J.K. Grogan, and H.S. Bassiouny, Flow-induced vein-wall vibration in an arteriovenous graft, ASME J. FluidStructures (to appear) (2005).

11. W.E. Lorensen and H. E. Cline, Marching cubes: A high resolution $3 d$ surface construction algorithm, SIGGRAPH '87: Proceedings of the 14th annual conference on Computer graphics and interactive techniques (New York, NY, USA), ACM Press, 1987, pp. 163-169.

12. F. Loth, N. Arslan, P. F. Fischer, C. D. Bertram, S. E. Lee, T. J. Royston, R. H. Song, W. E. Shaalan, and H. S. Bassiouny, Transitional flow at the venous anastomosis of an arteriovenous graft: Potential relationship with activation of the erk1/2 mechanotransduction pathway, ASME J. Biomech. Engr. 125 (2003), 49-61.

13. D.L. Marcum, Efficient generation of high quality unstructured surface and volume grids, October 2-5,2000.

14. T. S. Munson, Mesh shape-quality optimization using the inverse mean-ratio metric, Preprint ANL/MCS-P1136-0304, Argonne National Laboratory, Argonne, Illinois, 2004.

15. J. Shewchuk, Delaunay Refinement Mesh Generation, Ph.D. thesis, School of Computer Science, Carnegie Mellon University, Pittsburgh, Pennsylvania, May 1997, Available as Technical Report CMU-CS-97-137. 
16. J. Siek and A. Lumsdaine, Generic programming for high performance numerical linear algebra, http://www.osl.iu.edu/research/mtl.

17. G. Taubin, A signal processing approach to fair surface design, SIGGRAPH '95: Proceedings of the 22nd annual conference on Computer graphics and interactive techniques (New York, NY, USA), ACM Press, 1995, pp. 351-358.

18. G. Taubin, T. Zhang, and G. H. Golub, Optimal surface smoothing as filter design, ECCV '96: Proceedings of the 4th European Conference on Computer Vision-Volume I (London, UK), Springer-Verlag, 1996, pp. 283-292.

19. J.E. Thompson, Z.U.A. Warsi, and C. W. Mastin, Numerical grid generation, Elsevier Science Publisging Co., 1985. 